\title{
Submucosal Abscess of the Esophagus Caused by Piriform Sinus Fistula Treated with Transoral Video Laryngoscopic Surgery
}

\author{
Satoshi Koyama ${ }^{a}$ Kazunori Fujiwara $^{a}$ Tsuyoshi Morisaki ${ }^{b}$ \\ Takahiro Fukuhara $^{a}$ Katsuyuki Kawamoto ${ }^{a}$ Hiroya Kitano $^{a}$ \\ Hiromi Takeuchi ${ }^{a}$ \\ a Department of Otolaryngology Head and Neck Surgery, Tottori University Faculty of \\ Medicine, Yonago, and ${ }^{b}$ Center for Head and Neck Surgery, Kusatsu General Hospital, \\ Kusatsu, Japan
}

\section{Key Words}

Esophageal submucosal abscess · Piriform sinus fistula · Transoral video laryngoscopic surgery $\cdot$ Minimally invasive surgery

\begin{abstract}
Piriform sinus fistula (PSF) is a rare branchial anomaly that causes repetitive acute suppurative thyroiditis or deep neck abscess. The definitive treatment of PSF is open neck surgery. However, such surgery has a cosmetic problem and a high risk of recurrence. Furthermore, identifying the fistula is difficult due to previous repetitive infections. We report a case of esophageal submucosal abscess caused by PSF treated with endoscopic mucosal incision. The patient underwent transoral video laryngoscopic surgery (TOVS), and endoscopy as well as fluoroscopy revealed complete closure of PSF without any complication. TOVS is a novel surgical technique for the definitive treatment of PSF with esophageal submucosal abscess.
\end{abstract}

\section{Introduction}

Piriform sinus fistula (PSF) is a rare branchial anomaly derived from the congenital remnants of the third or fourth branchial cleft $[1,2]$. PSF occurs predominantly on the left side [3]. The clinical manifestations of PSF are repetitive acute suppurative thyroiditis or deep neck abscess, and it occurs in infancy or childhood in $80 \%$ of patients [4].

Submucosal abscess of the esophagus is extremely rare and has been reported so far in only three cases, due to tuberculosis and fish bone injury [5-7]. To our knowledge, esoph- 
Koyama et al.: Submucosal Abscess of the Esophagus Caused by Piriform Sinus Fistula Treated with Transoral Video Laryngoscopic Surgery

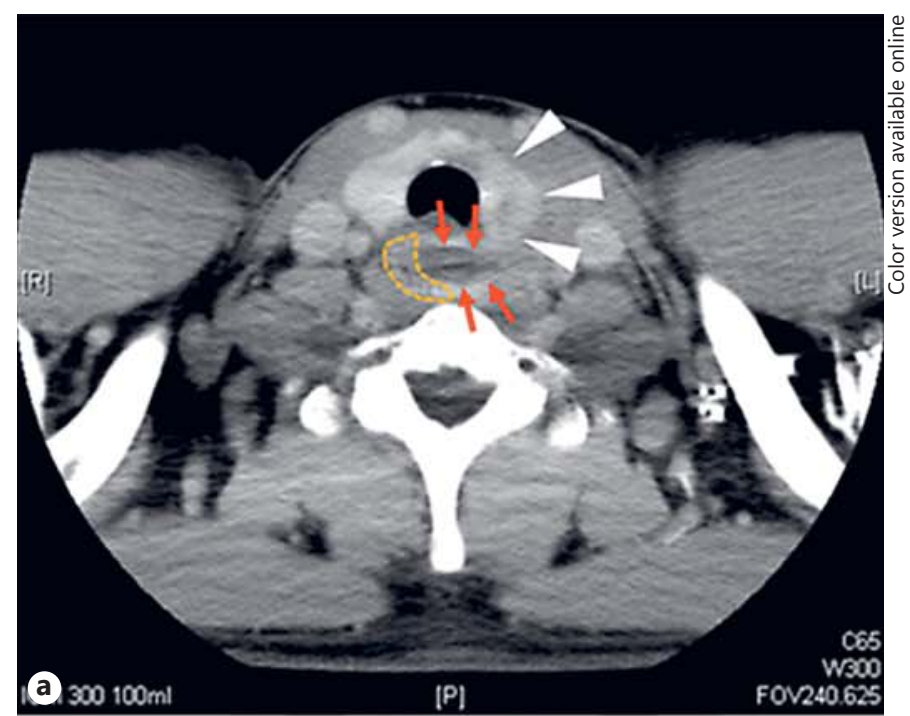

Fig. 1. a Contrast-enhanced CT (axial view). The dotted line indicates an original esophageal lumen and the arrows show an abscess in the submucosal layer of the esophageal wall. The white arrowheads show a low density in the left lobe of the thyroid. $\mathbf{b}$ Contrast-enhanced CT (coronal view). The dotted line shows an original esophageal lumen and the arrows show an abscess in the submucosal layer of the esophageal wall. c Endoscopic drainage of a submucosal esophageal abscess. We performed endoscopic mucosal incision and drainage of a submucosal esophageal abscess. White abscess was drained from the punctured esophageal mucosa.
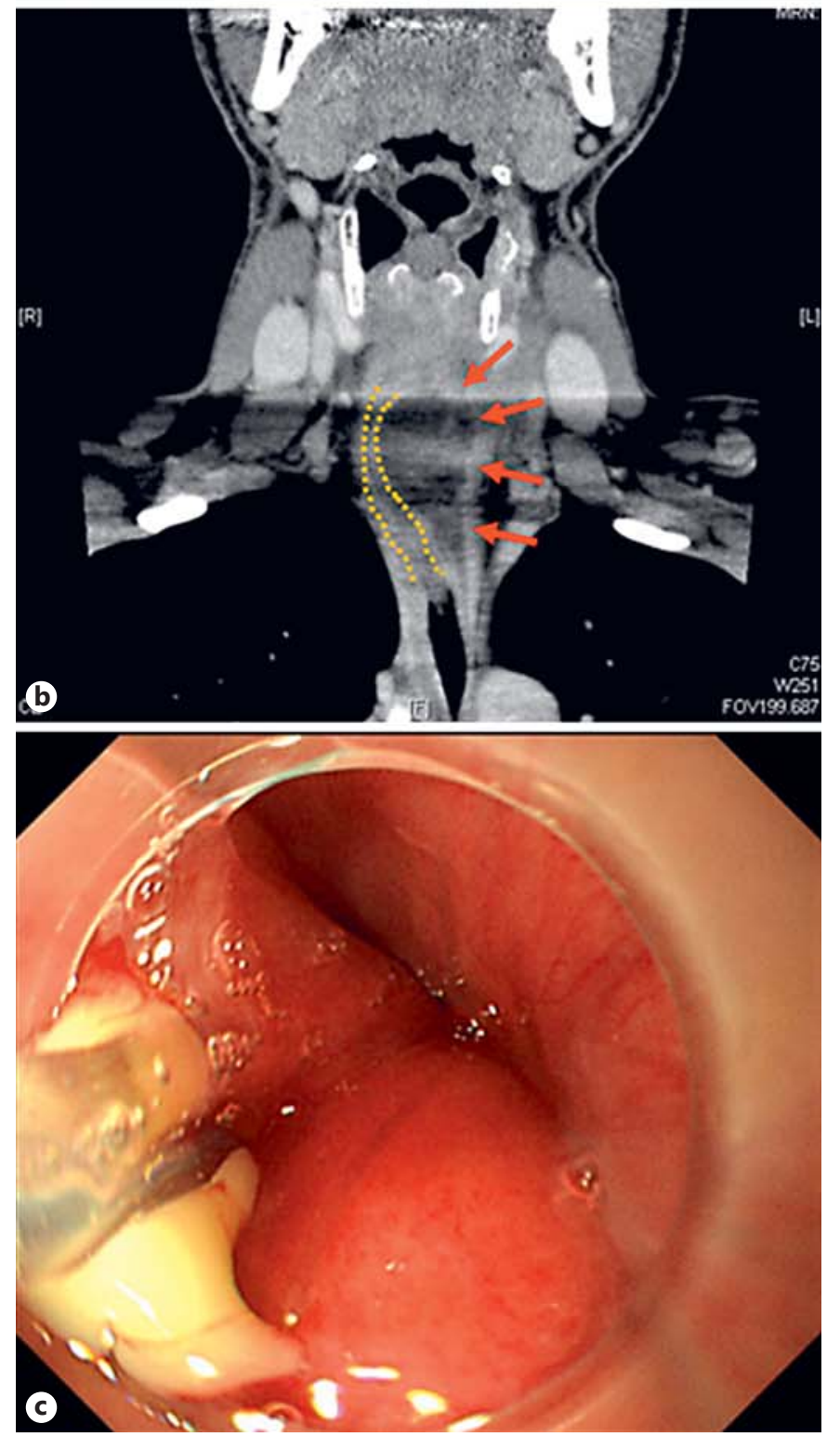
Koyama et al.: Submucosal Abscess of the Esophagus Caused by Piriform Sinus Fistula Treated with Transoral Video Laryngoscopic Surgery

Fig. 2. Video fluoroscopic examination revealed a PSF on the left side (arrow).

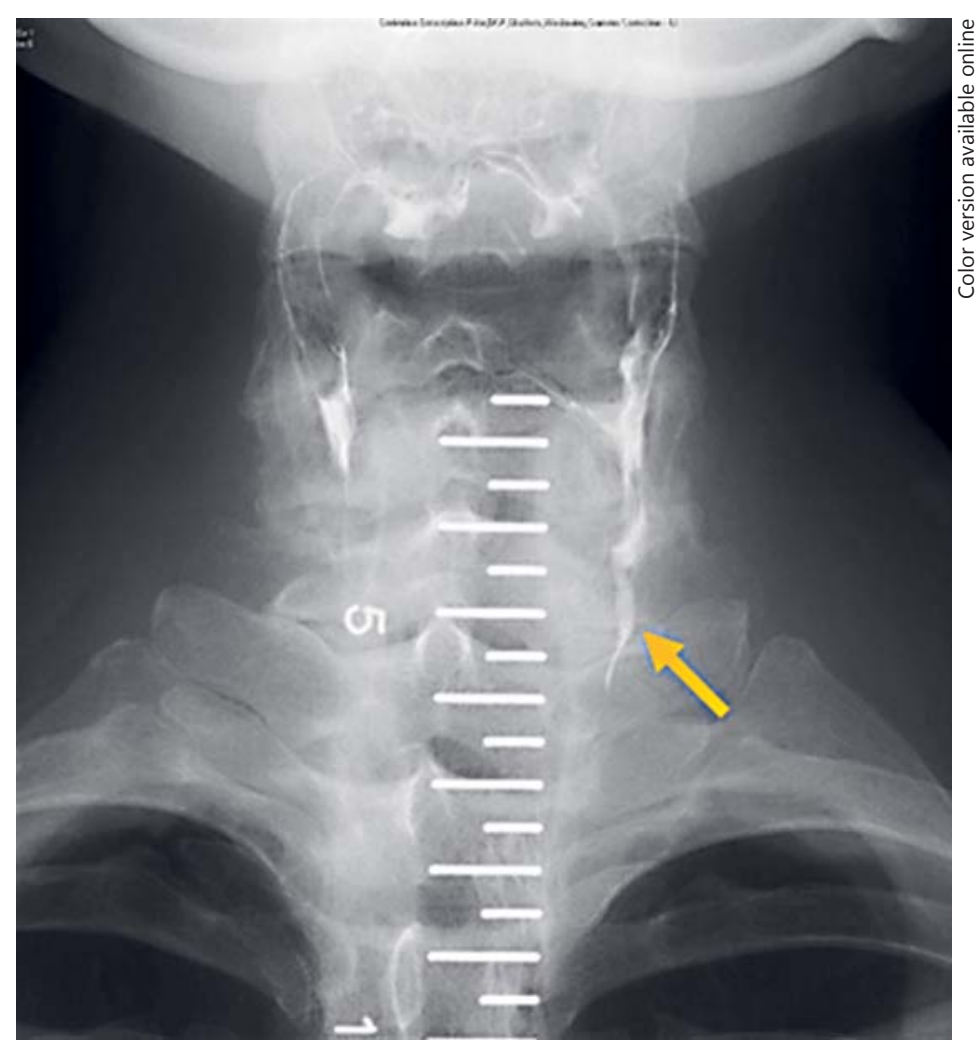

ageal submucosal abscess caused by deep neck infection has never been reported, and an abscess caused by a PSF in adults has also never been reported in the English literature.

The definitive treatment of PSF is open neck surgery with complete resection of the fistula with or without thyroid tissue. However, identification of the fistula is difficult due to the scarring caused by previous infections. Furthermore, the normal mucosal layers cannot be sutured during open neck surgery, which leads to a high risk of recurrence and cosmetic problems due to the neck incision. In addition, the surgical procedure carries a high risk of complications, such as injury to the recurrent laryngeal nerve [8, 9]. Thus, appropriate technical support is required to ensure the safety of the surgical procedure. In recent years, the use of transoral video laryngoscopic surgery (TOVS), which was developed as a surgical technique for superficial laryngopharyngeal cancer by Shiotani et al. [10] and Tanaka et al. [11], as the definitive treatment of PSF has been reported [12].

We report a case of esophageal submucosal abscess due to PSF treated with TOVS.

\section{Case Report}

A 41-year-old man was referred to our hospital with a suspected cervical esophageal submucosal abscess. Contrast-enhanced computed tomography (CT) revealed a heterogeneous low-density area in the left lobe of the thyroid with high density of the surrounding tissue and a homogeneous low-density area with ring enhancement in the submucosal layer of the esophageal wall from the lower neck to the upper mediastinum (fig. 1a, b). Laboratory examination showed elevation of the inflammation indicators, such as white blood cell count and C-reactive protein. However, thyroid hormone levels were within the normal limit. The diagnosis was an abscess in the submucosal layer of the esophagus with acute suppurative thyroiditis. Upper gastrointestinal endoscopy with the patient under local anesthesia revealed that the surface of the mucosa was edematous with abscess formation, and any cause of abscess, such as a fish bone, tumor, or diverticulum, was not found. Endoscopic mucosal incision was performed for drainage (fig. 1c), and the esophageal wall 


\begin{tabular}{l|l}
\hline ORL 2016;78:252-258 & \\
\hline DOI: 10.1159/000448589 & $\begin{array}{l}\text { @ 2016 S. Karger AG, Basel } \\
\text { www.karger.com/orl }\end{array}$ \\
\hline
\end{tabular}

Koyama et al.: Submucosal Abscess of the Esophagus Caused by Piriform Sinus Fistula Treated with Transoral Video Laryngoscopic Surgery
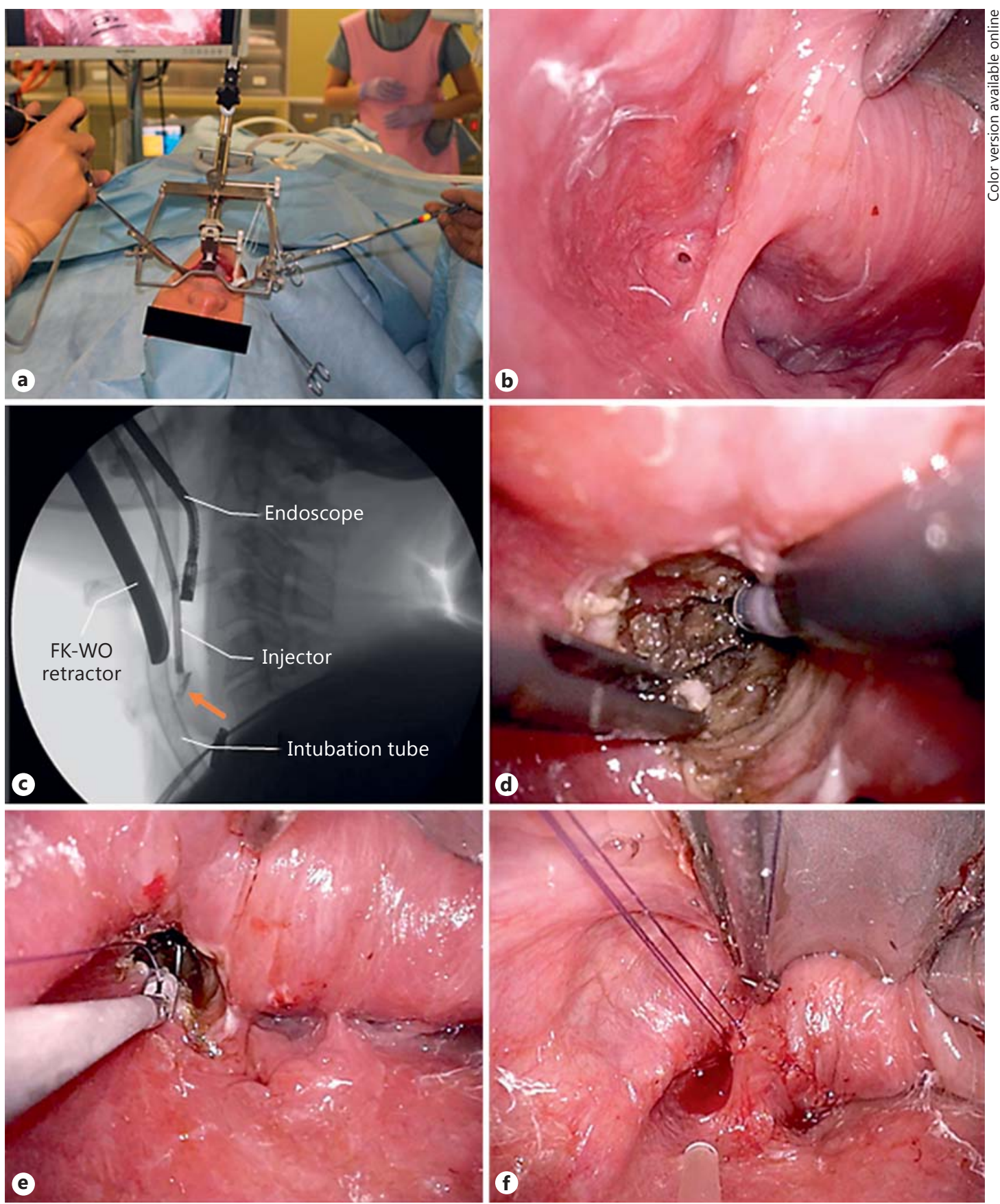

Fig. 3. a Intraoperative view. The patient was placed in the operative position with the FK-WO retractor inserted. The surgeon held the electrical surgical device (DualKnife), and the assistant held a rigid endoscope with a flexible tip. b Using the FK-WO retractor, the pharyngeal tract was opened, and endoscopy revealed the fistula orifice at the apex of the piriform sinus on the left. The larynx and recurrent laryngeal nerve were lifted ventrally, away from the surgical field. c Barium injection confirmed the fistula (arrow). Furthermore, the approximate depth and direction of the fistula were estimated. $\mathbf{d}$ The surgeon gently retracted the fistula with forceps and resected it with the other hand. e Normal hypopharyngeal mucosa was sutured with 4-0 PDS using a needle holder and knot tier. $\mathbf{f}$ The fistula was definitively resected and closed. 


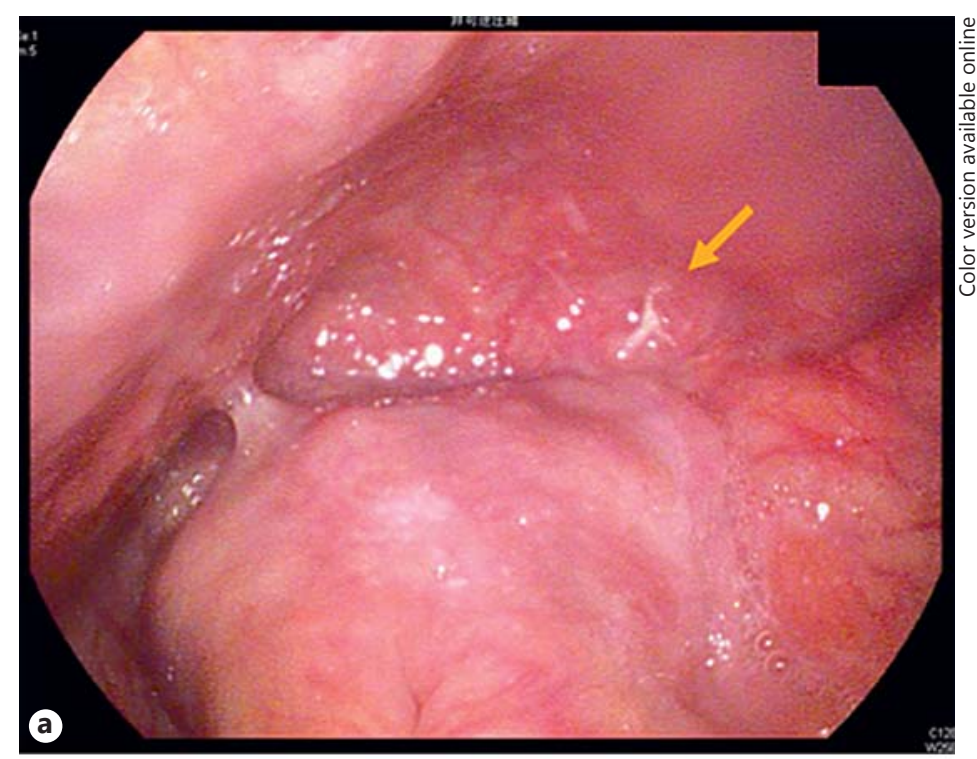

Fig. 4. a Endoscopic examination revealed an operative scar, and the fistula was completely closed. b Video fluoroscopy ensured closure of the fistula, and no recurrence was observed.

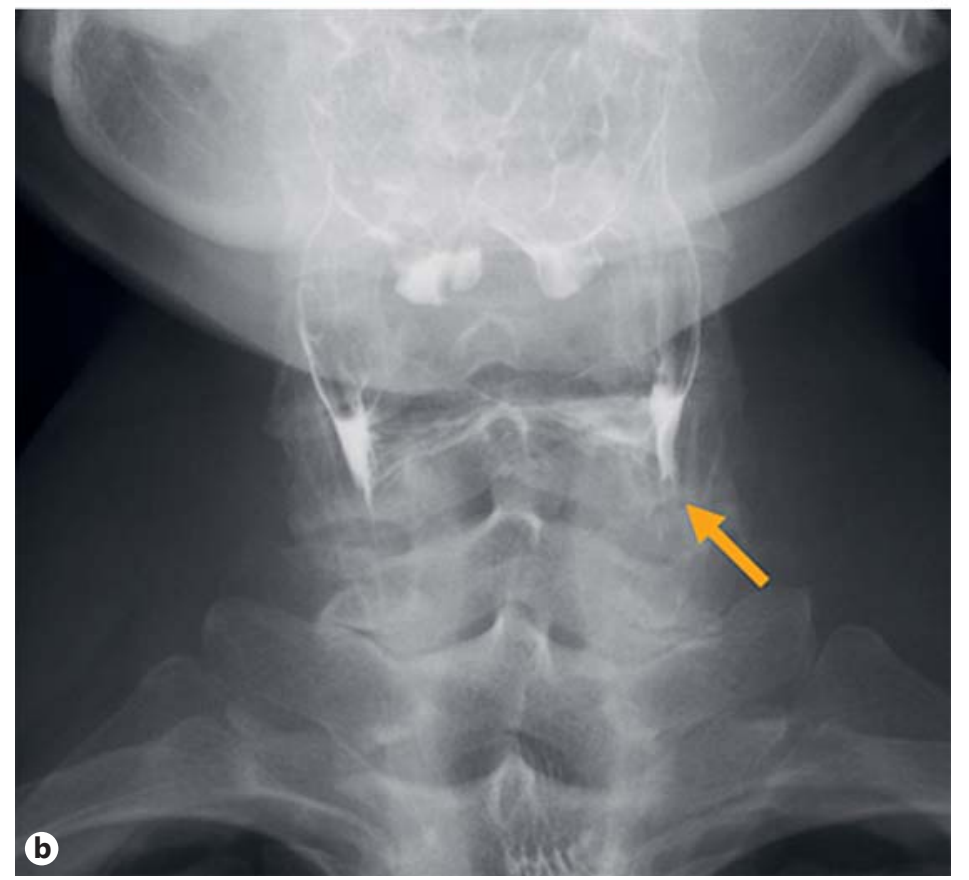

swelling resolved. Surgical drainage was performed and antibiotic treatment was administered, and no recurrence of the abscess occurred. Two weeks after drainage, video fluoroscopic examination of the hypopharynx, performed to investigate the cause of the esophageal submucosal abscess and acute suppurative thyroiditis, revealed PSF on the left side (fig. 2). We believed the inflammation of PSF was the cause of the esophageal submucosal abscess and acute suppurative thyroiditis.

Two surgical options for PSF were offered: open neck surgery or TOVS. The risks and benefits of each treatment were explained, and the patient chose transoral surgery. TOVS was performed with the patient under general anesthesia with transoral intubation. Immediately before the start of the operation, the patient swallowed a small amount of barium. An FK-WO retractor (Olympus, Tokyo, Japan) was inserted to expose the piriform sinus. An assistant held the high-resolution rigid endoscope with a flexible tip (Visera Elite OTV-S190 and LTF-S190-5; Olympus) and searched for the fistula orifice (fig. 3a) using X-ray fluoroscopy (BV Pulseral2; Philips Electronics Japan Ltd., Tokyo, Japan). Barium was stored in the fistula, and we found that the fistula orifice was at the apex of the piriform sinus (fig. 3b). Using fluoroscopy with barium injection to the fistula, we confirmed the fistula orifice and estimated the approximate depth (fig. 3c). We used a Dual- 
Koyama et al.: Submucosal Abscess of the Esophagus Caused by Piriform Sinus Fistula Treated with Transoral Video Laryngoscopic Surgery

$\mathrm{Knife}^{\mathrm{TM}}$ (Olympus) for the resection. At first, we made a circumferential incision around the fistula orifice. The submucosal layer was detached from the fistula and pursued toward the thyroid cartridge to the superficial hypopharyngeal constrictor muscle (fig. 3d). The fistula was definitively resected, and the remaining proximal hypopharyngeal mucosa was sutured with 4-0 PDS using a needle holder and knot tier (fig. 3e, f). Video fluoroscopic examination was performed 3 days after surgery and confirmed the closure of the PSF orifice. Oral intake was started. The patient was discharged from the hospital 7 days after surgery without any complications. At 1 month after surgery, endoscopic and video fluoroscopic examination revealed no evidence of recurrence (fig. $4 \mathrm{a}, \mathrm{b}$ ).

\section{Discussion}

PSF is known to cause repetitive acute suppurative thyroiditis or neck abscess. To our knowledge, we report the first case of esophageal submucosal abscess caused by PSF. PSF originated from the apex of the piriform sinus and coursed anteroinferiorly through the strap muscle layer, either beside or through the thyroid gland, and into the perithyroid space [2,13]. Thus, PSF is close to the cervical esophagus and there is a possibility that infection of PSF transmitted to the thyroid and esophagus resulted in submucosal abscess of the esophagus. In cases of esophageal submucosal abscess, we should consider the possibility of PSF in the differential diagnosis.

The conventional definitive treatment of PSF is open neck surgery and complete resection of the fistula. However, open neck surgery has cosmetic problems due to the skin incision. The complicated fibrosis due to repetitive PSF infection makes identification of the fistula tract challenging even for experienced surgeons. Consequently, there is a high risk of surgical complications, such as recurrent laryngeal nerve injury. Furthermore, there is a high incidence of disease recurrence because of the vulnerability of the sutured fistula tissue.

Recently, transoral chemocauterization or $\mathrm{CO} 2$ laser ablation of the fistula's internal orifice has been reported as a potential minimally invasive surgical treatment of PSF [14]. Transoral endoscopic chemocauterization of the fistula's internal orifice also has been reported in the past decade $[15,16]$. These low invasive surgical procedures do not need any skin incision on the neck and have less serious complications and postoperative pain. Nevertheless, patients often experience recurrent infection due to the unresected fistula duct, and closure of the orifice depends on the cauterization and not the mucosal layer. Thus, this procedure is considered only for vulnerable patients undergoing open neck surgery, such as those with low ADL scores or low performance status and the elderly.

Kamide etal. [12] reported on TOVS for definitive resection of a fistula from the pharyngeal tract in 2015. With this procedure, the greater part of the fistula could be resected and normal mucosal layers sutured, ensuring closure of the fistula orifice without any serious complications and skin incision. The fistula orifice could be identified from the pharyngeal tract, and performance of the procedure did not depend on the previous infection and scarring around the fistula tract. However, this procedure has several problems: identification of the fistula orifice is not as easy in several cases, and there is the risk of recurrent laryngeal nerve injury. Furthermore, this procedure could not be applied in patients with trismus.

A preoperative barium swallow has been reported to assist in finding the fistula in the open neck surgical field [17]. In our patient, a barium swallow performed immediately before the operation and fluoroscopic examination during the operation assisted in locating the fistula orifice. Furthermore, video fluoroscopy was useful to locate the PSF orifice during the operation and aided in real-time estimation of the fistula length and direction. High-resonance endoscopy revealed the fistula clearly, and the surgeon could pursue and resect the PSF without any stress.

TOVS requires the FK-WO retractor to expose the piriform sinus; this moves the larynx and the recurrent laryngeal nerve ventrally, away from the piriform sinus. Thus, the incidence 
Koyama et al.: Submucosal Abscess of the Esophagus Caused by Piriform Sinus Fistula Treated with Transoral Video Laryngoscopic Surgery

of nerve injury was reduced. Furthermore, intraoperative electromyographic monitoring is recommended to reduce the potential risk of nerve injury; in our patient, we used this technique to confirm that the recurrent laryngeal nerve was preserved.

At 1 month after the operation, endoscopy and video fluoroscopy revealed no disease recurrence. TOVS is a safe, easy, and reliable definitive treatment of PSF.

\section{Acknowledgment}

We gratefully acknowledge the work of past and present members of our department.

\section{Statement of Ethics}

The protocol of the investigation has been approved by the Institutional Review Board at Tottori University Hospital (No. 1603A154).

\section{Disclosure Statement}

The authors have no conflicts of interest to disclose.

\section{References}

1 Edmonds JL, Girod DA, Woodroof JM, Bruegger DE: Third branchial anomalies. Avoiding recurrences. Arch Otolaryngol Head Neck Surg 1997;123:438-441.

2 Godin MS, Kearns DB, Pransky SM, Seid AB, Wilson DB: Fourth branchial pouch sinus: principles of diagnosis and management. Laryngoscope 1990;100:174-178.

3 Takai SI, Miyauchi A, Matsuzuka F, Kuma K, Kosaki G: Internal fistula as a route of infection in acute suppurative thyroiditis. Lancet 1979;313:751-752.

4 Miyauchi A, Matsuzuka F, Takai S, Kuma K, Kosaki G: Piriform sinus fistula. A route of infection in acute suppurative thyroiditis. Arch Surg 1981;116:66-69.

5 Eroğlu A, Kürkçüoğlu C, Karaoğlanoğlu N, Yilmaz O, Gürsan N: Esophageal tuberculosis abscess: an unusual cause of dysphagia. Dis Esophagus 2002;15:93-95.

6 Lee KH, Kim HJ, Kim KH, Kim HG: Esophageal tuberculosis manifesting as submucosal abscess. AJR Am J Roentgenol 2003;180:1482-1483.

7 Takeno S, Moroga T, Ono K, Kawahara K, Hirano T, Moriyama M, et al: Endoscopic mucosal incision for successful treatment of submucosal abscess extending the full length of the esophagus due to fish bone: report of a case. Esophagus 2015;12:199-202.

8 James A, Stewart C, Warrick P, Tzifa C, Forte V: Branchial sinus of the piriform fossa: reappraisal of third and fourth branchial anomalies. Laryngoscope 2007;117:1920-1924.

9 Sheng Q, Lv Z, Xiao X, Zheng S, Huang Y, Huang X, et al: Diagnosis and management of piriform sinus fistula: experience in 48 cases. J Pediatr Surg 2014;49:455-459.

10 Shiotani A, Tomifuji M, Araki K, Yamashita T: Transoral videolaryngoscopic surgery for en bloc resection of supraglottic and hypopharyngeal cancers. Otolaryngol Head Neck Surg 2011;144:288-289.

11 Tanaka Y, Tomifuji M, Suzuki H, Yamashita T, Araki K, Shiotani A: Transoral videolaryngoscopic surgery with a navigation system for excision of a metastatic retropharyngeal lymph node. ORL 2014;76:357-363.

12 Kamide D, Tomifuji M, Shiotani A, Utsunomiya K, Yamashita T, Araki K, et al: Minimally invasive surgery for piriform sinus fistula by transoral videolaryngoscopic surgery. Am J Otolaryngol 2015;36:601-605.

13 Park SW, Han MH, Sung MH, Kim IO, Kim KH, Chang KH, et al: Neck infection associated with piriform sinus fistula: imaging findings. Am J Neuroradiol 2000;21:817-822.

14 Kim K, Sung M, Koh T, Oh S, Kim I: Piriform sinus fistula: management with chemocauterization of the internal opening. Ann Otol Rhinol Laryngol 2000;109:452-456.

15 Cha W, Cho SW, Hah HJ, Kwon TK, Sung MW, Kim KH: Chemocauterization of the internal opening with trichloroacetic acid as first-line treatment for piriform sinus fistula. Head Neck 2013;35:431-435.

16 Zhang J, Huang S, Li H, Chen H, Gu L: Relapsing suppurative neck abscess after chemocauterization of piriform sinus fistula. Clin Imaging 2012;36:826-828.

17 Madana J, Yolmo D, Kalaiarasi R, Gopalakrishnan S, Saxena SK, Krishnapriya S: Recurrent neck infection with branchial arch fistula in children. Int J Pediatr Otorhinolaryngol 2011;75:1181-1185. 